\title{
Improvement of Brain Tissue Segmentation Using Information Fusion Approach
}

\author{
Lamiche Chaabane \\ Department of Computer Science \\ University of M'sila \\ Algeria
}

\author{
Moussaoui Abdelouahab \\ Department of Computer Science \\ University of Setif \\ Algeria
}

\begin{abstract}
The fusion of information is a domain of research in full effervescence these last years. Because of increasing of the diversity techniques of images acquisitions, the applications of medical images segmentation, in which we are interested, necessitate most of the time to carry out the fusion of various data sources to have information with high quality. In this paper we propose a system of data fusion through the framework of the possibility theory adapted for the segmentation of MR images. The fusion process is divided into three steps : fuzzy tissue maps are first computed on all images using Fuzzy C- Means algorithm. Fusion is then achieved for all tissues with a fusion operator. Applications on a brain model show very promising results on simulated data and a great concordance between the true segmentation and the proposed system.
\end{abstract}

Keywords- Information fusion; possibility theory; segmentation; FCM; MR images.

\section{INTRODUCTION}

Recent technical advances have led to the multiplication of imaging systems, which are often used for observing a phenomenon from different points of view. They provide a large amount of information that must a whole in order to draw correct conclusions. This development has made data fusion in image processing an important step, now well recognized, in modern multi source image analysis. In medical imaging in particular the clinician may use images issued from different sources, each of them highlighting specific properties of tissues and pathologies. They may be images acquired with a single imaging technique using different acquisition parameters (for instance multi echo MRI), or images were obtained from several imaging techniques (for instance anatomical MR imaging combined with functional PET imaging). The association of such images allows the medical expert to confirm and to complete his diagnosis because it can arrive as none the images available contains a sufficient information separately. In most data fusion problems, the images to be combined are partly redundant as they represent the same scene, and partly complementary as they may highlight different characteristics [1].

Typically, none of the images provide a completely decisive and reliable information. In addition, the information is often imprecise and uncertain, and these characteristics are inherent to the images; due to observed phenomenon, sensors, numerical reconstruction algorithms and resolution etc. The aim of data fusion techniques is therefore to improve the decision by increasing the amount of global information, while decreasing its imprecision and uncertainty by making us of redundancy and complementarities [1].

Some mathematical models were discussed in the literature for the modelization of both uncertainty and imprecision. Traditionally probabilities theory was the primary model used to deal with uncertainty problems, but they suffer from drawbacks which are still a matter of discussion. Whereas the Dempster-Shafer theory also allows to representing these two natures of information using functions of mass but the set of operators used by this theory in fusion step is very restricted. Alternative to this approach is the possibility theory where uncertainty and imprecision are easily modeled, in this article we will focus on this last one for two essential reasons : this theory allows to combining information coming from various sources by the use a wide range of available combination operators. In addition, this theory seems to us the most adapted to the considered problem in the modeling step [1][2].

We present in this article, a fuzzy information fusion framework for the automatic segmentation of human brain tissues using T2- weighted (T2) and proton density (PD) images. This framework consists of the computation of fuzzy tissue maps in both images by means of Fuzzy C-Means algorithm, the creation of fuzzy maps by a combination operator and a segmented image is computed in decision step.

The organization of the paper as follows : In section II, some previous related works are presented. In section III, we briefly outline the principals of possibility theory reasoning. Section IV discusses the architecture of the fusion system. Steps of fusion in possibility theory are explained in Section V. In section VI the traditional FCM algorithm is briefly reviewed. Section VII presents our proposed approach. Some experiment results using two routing MR sequences T2 and PD feature images are shown in section VIII and section IX contains conclusions and addresses future works.

\section{RELATED WORKS}

Many works have been done in the field of fuzzy information fusion in the literature. A brief review of some of them is presented in this section. Waltz [6] presented three basic levels of image data fusion : pixel level, feature level and decision level, which correspond to three processing architectures. I. Bloch [1] have outlined some features of Dempster-Shafer evidence theory, which can very useful for 
medical image fusion for classification, segmentation or recognition purposes. Examples were provided to show its ability to take into account a large variety of situations. Registration-based methods are considered as pixel-level fusion, such as MRI-PET (position emission tomography) data fusion [7]. Some techniques of knowledge-based segmentation can be considered as the feature-level fusion such as the methods proposed in [11].

Some belief functions, uncertainty theory, Dempster-Shafer theory are often used for decision-level fusion such as in [9]. In [12], I. Bloch proposed an unified framework of information fusion in the medical field based on the fuzzy sets, allow to represent and to process the numerical data as well as symbolic systems, the fuzzy sets theory is applied to three levels: at the low level to treat the basic numerical information contained in the images, as well as possible ambiguity between the classes; on the level object, to represent objects or structures in the images such as a fuzzy objects. at the higher level, to take into account a structural information and some characteristics as the distance, adjacency, and the relative position between objects.

V. Barra and J. Y. Boire [4] have described a general framework of the fusion of anatomical and functional medical images. The aim of their work is to fuse anatomical and functional information coming from medical imaging, the fusion process is performed in possibilistic logic frame, which allows for the management of uncertainty and imprecision inherent to the images. They particularly focus on the aggregation step with the introduction of a new class of operators based on information theory and the whole process is finally illustrated in two clinical cases: the study of Alzheimer's disease by MR/SPECT fusion and the study of epilepsy with MR/PET/SPECT. The obtained results was very encouraging.

V. Barra and J. Y. Boire [10] proposed a new scheme of information fusion to segment intern cerebral structures. The information is provided by MR images and expert knowledge, and consists of constitution, morphological and topological characteristics of tissues. The fusion of multimodality images is used in [8]. In [3], the authors have presented a framework of fuzzy information fusion to automatically segment tumor areas of human brain from multispectral magnetic resonance imaging (MRI) such as T1-weighted, T2-weighted and proton density (PD) images; in this approach three fuzzy models are introduced to represent tumor features for different MR image sequences. They allow to create corresponding fuzzy feature space of tumor. All the t-norm or fuzzy intersection operators can be used as fusion operators for this fuzzy features. the geometric mean is chosen using experiments allowing us to take correctly into account the three fuzzy spaces in a simple way. The fuzzy region growing is used to improve the fused result.

Maria del C and al [5] proposed a new multispectral MRI data fusion technique for white matter lesion segmentation, in that a method is described and comparison with thresholding in FLAIR images is illustrated.

\section{THE POSSIBILITy THEORY}

Possibilistic logic was introduced by Zadeh (1978) following its former works in fuzzy logic (Zadeh, 1965) in order to simultaneously represent imprecise and uncertain knowledge. In fuzzy set theory, a fuzzy measure is a representation of the uncertainty, giving for each subset $Y$ of the universe of discourse $X$ a coefficient in [0,1] assessing the degree of certitude for the realization of the event $Y$. In possibilistic logic, this fuzzy measure is modeled as a measure of possibility $\Pi$ satisfying:

$$
\begin{aligned}
& \Pi(X)=1 \quad \text { et } \quad \Pi(\phi)=0 \\
& \left(\forall\left(Y_{i}\right)\right) \Pi\left(\cup_{i} Y_{i}\right)=\operatorname{Sup}_{i} \Pi\left(Y_{i}\right)
\end{aligned}
$$

An event $\mathrm{Y}$ is completely possible if $\Pi(Y)=1$ and is impossible if $\Pi(Y)=0$. Zadeh showed that $\Pi$ could completely be defined from the assessment of the certitude on each singleton of $X$. Such a definition relies on the definition of a distribution of possibility $\pi$ satisfying :

$$
\begin{aligned}
& \pi: X \rightarrow[0,1] \\
& x \rightarrow \pi(x) / \operatorname{Sup}_{x}\{\pi(x)=1\}
\end{aligned}
$$

Fuzzy sets $\mathrm{F}$ can then be represented by distributions of possibility, from the definition of their characteristic function $\mu_{F}:$

$(\forall x \in X) \mu_{F}(x)=\pi(x)$

Distributions of possibility can mathematically be related to probabilities, and they moreover offer the capability to declare the ignorance about an event. Considering such an event $\mathrm{A}$ (e.g., voxel $\mathrm{v}$ belongs to tissue $\mathrm{T}$, (where $\mathrm{v}$ is at the interface between two tissues), the probabilities would assign $P(A)=P(\bar{A})=0.5$, whereas the possibility theory allows fully possible $\Pi(A)=\Pi(\bar{A})=1$. We chose to model all the information using distributions of possibility, and equivalently we represented this information using fuzzy sets [18].

The literature classically distinguishes three modes for combination of uncertainty and imprecise information in a possibility theory framework [23] :

The conjunction: gather the operators of t-norms (fuzzy intersection), this mode of combination must be used if measurements are coherent, i.e. without conflict.

The compromise: gather the median operator and some average operators, it must be used when measurements are in partial conflict.

The Disjunction: gather the operators of t-conorms (fuzzy union), it must be used when measurements are in disaccord, i.e. in severe conflict.

In introduction, we underlined the inopportunity to combining information in a fixed mode: if observations are in 
accord, it is legitimate to combine them in a conjunctive mode or compromise in order to extract a more relevant information. But if a serious conflict appears, it is better to combining in a disjunctive mode. For example, if two measurements of the same parameter prove completely different, it is not judicious to make an average of it, better is worth to say than one or the other is true [24].

\section{THE FUSION PROCESS AND TYPE OF ARCHITECTURES}

A general information fusion problem can be stated in the following terms: given $l$ sources $S_{l}, S_{2}, \ldots S_{l}$ representing heterogeneous data on the observed phenomenon, take a decision $d_{i}$ on an element $x$, where $x$ is higher level object extracted from information, and Di belongs to a decision space $D=\left\{d_{1}, d_{2}, d_{3}, \ldots, d_{n}\right\}$ (or set of hypotheses). In numerical fusion methods, the information relating $x$ to each possible decision $d_{i}$ according to each source $S_{j}$ is represented as a number $M_{i j}$ having different properties and different meanings depending on the mathematical fusion framework. In the centralized scheme, the measures related to each possible decision $i$ and provided by all sources are combined in a global evaluation of this decision, taking the form, for each $i: M i=F\left(M_{i 1}, M_{i 2}, M_{i 3}\right.$, ..., $M_{i n}$ ), where $F$ is a fusion operator. Then a decision is taken from the set of $M_{i}, 1 \leq i \leq n$. in this scheme, no intermediate decision is taken and the final decision is issued at the end of the processing chain. In decentralized scheme decisions at intermediate steps are taken with partial information only, which usually require a difficult control or arbitration step to diminish contradictions and conflicts [2][4].

The three-steps fusion can be therefore described as :

- Modeling of information in a common theoretical frame to manage vague, ambiguous knowledge and information imperfection. In addition, in this step the $M_{i j}$ values are estimated according to the chosen mathematical framework.

- Combination : the information is then aggregated with a fusion operator $F$. This operator must affirm redundancy and manage the complementarities and conflicts.

- Decision : it is the ultimate step of the fusion, which makes it possible to pass from information provided by the sources to the choice of a decision $d_{i}$.

\section{FUSION IN POSSIBILITY THEORY}

\section{A. Modeling Step}

In the framework of possibility theory and fuzzy sets [13][14[15], the $M_{i j}$ 's represent membership degrees to a fuzzy set or possibility distribution $\pi$, taking the form for each decision $d_{i}$ and source $S_{i}: M_{i j}=\pi_{j}\left(d_{i}\right)$.

\section{B. Fusion step}

For the aggregation step in the fusion process, the advantages of possibility theory rely in the variety of combination operators, which must affirm redundancy and manage the complementarities. And may deal with heterogeneous information [16][17][18]. It is particular interest to note that, unlike other data fusion theories like Bayesian or Dempster-Shafer combination, possibility theory provides a great flexibility in the choice of the operator, that can be adapted to any situation at hand [2].

\section{Decision step}

Is usually taken from maximum of memberships values after the aggregation step. Many constraints can be added to this decision, typically for checking for the reliability of the decision (is he obtained value high enough?) or for the discrimination power of he fusion (is the difference between the two highest values high enough ?) [2].

\section{THE FCM AlgORITHM CluStering}

Clustering is a process of finding groups in unlabeled dataset based on a similarity measure between the data patterns (elements) [12]. A cluster contains similar patterns placed together. The fuzzy clustering technique generates fuzzy partitions of the data instead of hard partitions. Therefore, data patterns may belong to several clusters, having different membership values with different clusters. The membership value of data pattern to a cluster denotes similarity between the given data pattern to the cluster. Given a set of $N$ data patterns $X=\left\{x_{1}, x_{2}, x_{3}, \ldots, x_{n}\right\}$ the Fuzzy C-Means (FCM) clustering algorithm minimizes the objective function [26][27]:

$$
J(B, U, X)=\sum_{i=1}^{C} \sum_{j=1}^{N} u_{i j}^{m} d^{2}\left(x_{j}, b_{i}\right)
$$

Where $x_{j}$ is the $j$-th P-dimensional data vector, $b_{i}$ is the center of cluster $i, u_{i j}$ is the degree of membership of $x_{j}$ in the $j$ th cluster, $m$ is the weighting exponent $d^{2}\left(x_{j}, b_{i}\right)$ is the Euclidean distance between data $x_{j}$ and cluster center $b_{i}$.

The minimization of objective function $J(B, U, X)$ can be brought by an iterative process in which updating of membership $u_{i j}$ and the cluster centers are done for each iteration.

$$
\begin{gathered}
u_{i j}=\left[\sum_{k=1}^{C}\left(\frac{d^{2}\left(x_{j}, b_{i}\right)}{d^{2}\left(x_{j}, b_{k}\right)}\right)^{2 /(m-1)}\right]^{-1} . \\
b_{i}=\frac{\sum_{k=1}^{N} u_{i j}^{m} x_{k}}{\sum_{k=1}^{N} u_{i k}^{m}} .
\end{gathered}
$$

Where :

$$
\begin{gathered}
\forall i \in\{1 . . C\}, \quad \forall j \in\{1 . . N\} \quad\left\{\begin{array}{c}
u_{i j} \in[0,1] \\
0<\sum_{i=1}^{N} u_{i j}<N
\end{array} .\right. \\
\forall j \in\{1 . . N\} \quad \sum_{i=1}^{C} u_{i j}=1 .
\end{gathered}
$$


The algorithm of the FCM consists then of the reiterated application of (2) and (3) until stability of the solutions.

\section{PROPOSED METHOD}

In this section we propose a framework of data fusion based on the possibility theory which allows the segmentation of MR images. The operation is limited to the fusion of $\mathrm{T} 2$ and PD images. Then information to combine are thus homogeneous and the scheme of our proposed fusion system as shown in figure 1 below:

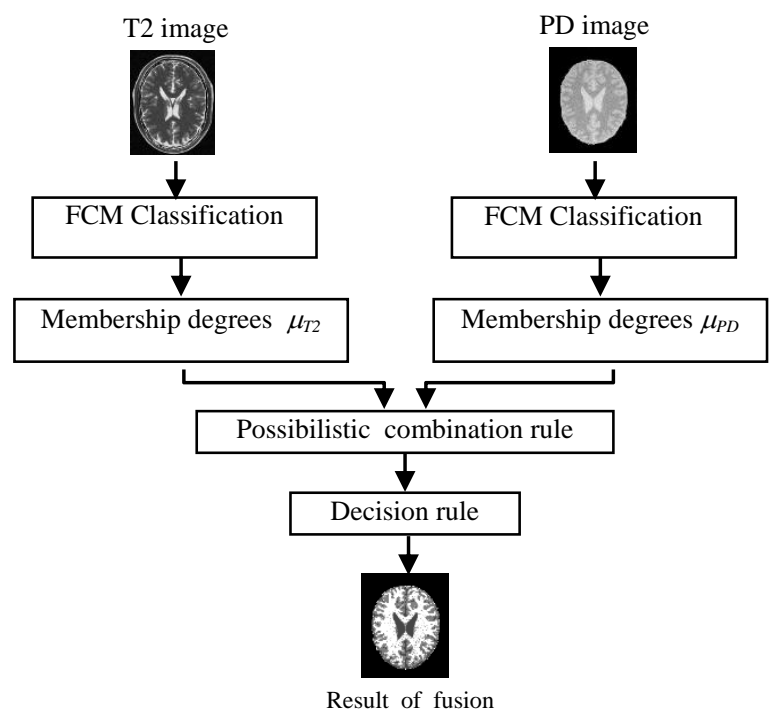

Figure 1. Scheme of the proposed fusion system.

If it is supposed that these images are registered, our approach of fusion consists of three steps:

\section{A. Modeling of the data}

In this phase the choice of the fuzzy framework is retained to modeling information resulting from the various images. More precisely, MR images are segmented in $C=4$ classes using the FCM algorithm described in section VI. For each MR image $I, C$ distributions of possibility $\pi_{T}^{I}, 1 \leq T \leq C$ are then obtained, and are represented by memberships of the pixels to the classes.

\section{B. Combination}

The aggregation step is most fundamental for a relevant exploitation of information resulting from the images $I_{T 2}, I_{P D}$. The operator must combine for a given tissue $T$, the distributions of possibility $\pi^{T 2}$ and $\pi^{P D}$, by underlining the redundancies and managing ambiguities and complementarities between the T2-weighted and proton density images.

1) Choice of an operator: One of the strengths of the possibility theory is to propose a wide range of operators for the combination of memberships. I. Bloch [20] classified these operators not only according to their severe (conjunctive) or cautious (disjunctive) nature but also with respect to their context-based behavior. Three classes were thus defined:
- Context independent and constant behavior operators (CICB);

- Context independent and variable behavior operators (CIVB);

- Context dependent operators (CD).

For our T2/PD fusion, we chose a (CICB) class of combination operators because in the medical context, both images were supposed to be almost everywhere concordant, except near boundaries between tissues and in pathologic areas [20]. Three operators (minimum, maximum, and arithmetic mean) of this class who does not need any parameter were tested related to the fusion of $\mathrm{MR}$ images acquired in weighting T2, PD. They were carried out on a range of 70 slices of Brain1320 volume of Brainweb ${ }^{1}$ If $\pi_{T}^{T 2}, \pi_{T}^{P D}$ are the possibility distributions of tissue $\mathrm{T}$ derived from $\mathrm{T} 2$ and $\mathrm{PD}$ maps, then the fused possibility as defined for any gray level $v$ as :

The minimum operator: $\pi_{T}(v)=\operatorname{Min}\left(\pi_{T}^{T 2}(v), \pi_{T}^{P D}(v)\right)$

The maximum operator: $\pi_{T}(v)=\operatorname{Max}\left(\pi_{T}^{T 2}(v), \pi_{T}^{P D}(v)\right)$

The arithmetic mean operator: $\pi_{T}(v)=\left(\pi_{T}^{T 2}(v)+\pi_{T}^{P D}(v)\right) / 2$

These operators are compared with the reference result using the coefficient $\mathrm{DSC}^{2}$. Which measures the overlap between two segmentations $\mathrm{S} 1$ and $\mathrm{S} 2$. It is defined as:

$$
\operatorname{DSC}(S 1, S 2)=2 \cdot \operatorname{card}(S 1 \cap S 2) / \operatorname{card}(S 1+S 2)
$$

The results of these tests are shown on figure 2:

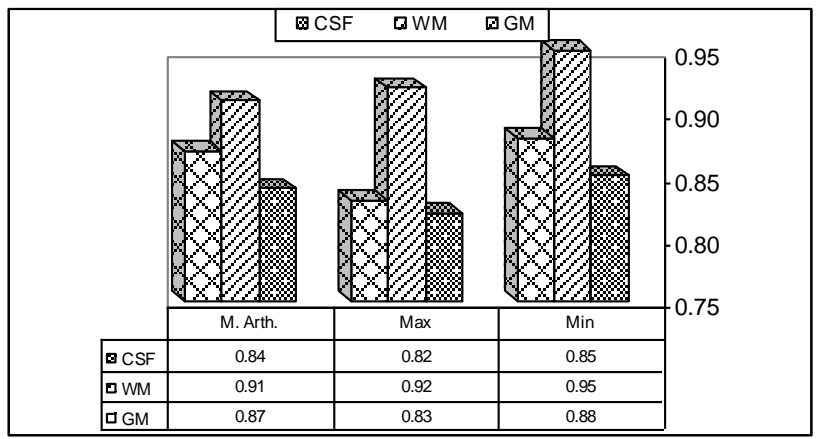

Figure 2. Comparison of the operators by the DSC measurement.

The results drawn up in the figure 2 show the predominance of the minimum operator compared to the maximum operator and the arithmetic mean operator. Thus we will retaining this operator for our study.

\section{Decision}

A segmented image was finally computed using all maps of different tissues $T, 1 \leq T \leq C$. So certain theories make it possible to consider several types of decision, the theory of the possibilities proposes only the rule of the maximum of

\footnotetext{
${ }^{1}$ http://www.bic.mni.mcgill.ca/brainweb/

${ }^{2}$ Dice Similarity Coefficient
} 
possibility. We thus retain this one and assign each pixel to the tissue for which it has the greatest membership.

The general algorithm of our system is .

\begin{tabular}{l}
\hline General algorithm \\
\hline Modeling of the image \\
For i in $\{\mathrm{T} 2, \mathrm{PD}\}$ do \\
FCM (i) $\quad\{$ Computation of membership degrees \\
$\quad$ for both images $\}$
\end{tabular}

\section{End For \\ Fusion \\ Possibilistic fusion $\{$ Between each class of T2 image and the same one of PD image $\}$}

Decision

Segmented image

It should be noted that the stability of our system depend to the stability of the algorithm used in the modeling step[26].

\section{RESULTS AND DISCCUSION}

Since the ground truth of segmentation for real MR images is not usually available, it is impossible to evaluate the segmentation performance quantitatively, but only visually. However, Brainweb provides a simulated brain database (SBD) including a set of realistic MRI data volumes produced by an MRI simulator. These data enable us to evaluate the performance of various image analysis methods in a setting where the truth is known [30][31][32].

to have tests under realistic conditions, three volumes were generated with a thickness of $1 \mathrm{~mm}$ and a level of noise of $0 \%$, $3 \%$ and $5 \%$. We fixed at $20 \%$ the parameter of heterogeneity.

The results of each step of fusion are presented on a noisy 90 th brain only slice is shown in figure 3 . This noisy slice was segmented into four clusters: background, CSF, white matter, and gray matter using FCM algorithm, however the background was neglected from the viewing results.

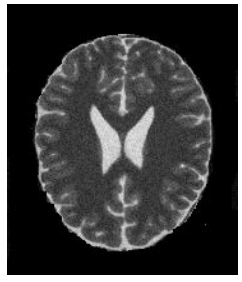

Simulated MR T2 image

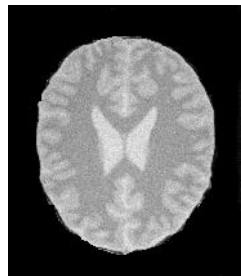

Simulated MR PD image (a) (b)
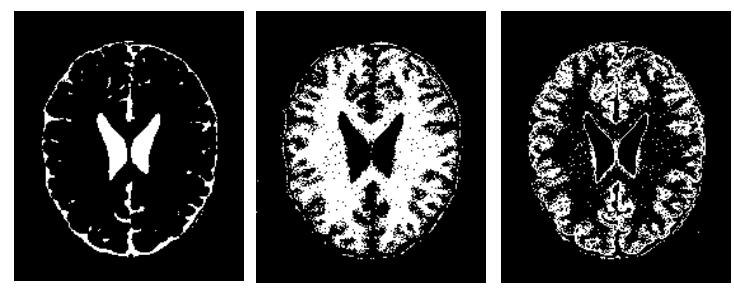

(c)
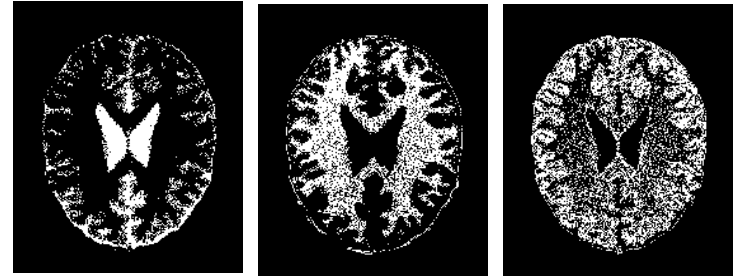

(d)
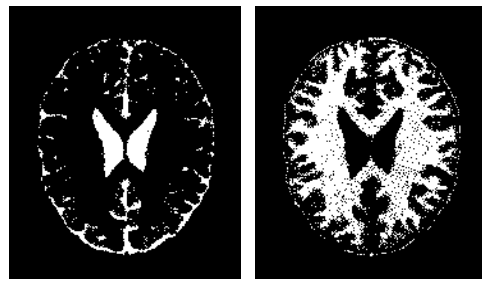

WM

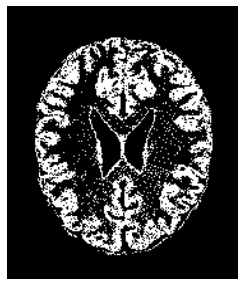

GM

Figure 3. (a) Simulated T2, PD images illustrate the fusion. (b) Maps of CSF, WM and GM obtained by FCM for T2 image. (c) Maps of CSF, WM and GM obtained by FCM for PD image . (d) Maps of CSF, WM and GM obtained by proposed system.

The results of final segmentation are shown in figure 4 below.

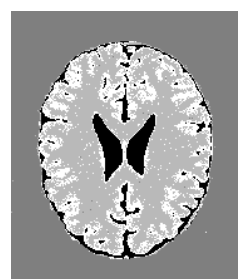

(a)

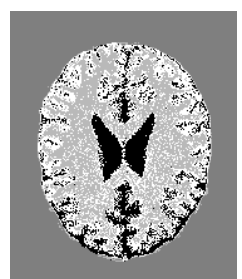

(b)

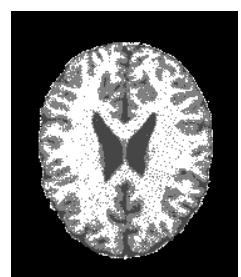

(c)

Figure 4. Segmentation results. (a) T2 segmented by FCM (b) PD segmented by FCM, (c) Image of fusion

The CSF map of PD image is improved significantly by fusion within the noise levels $0 \%-5 \%$.

The WM fused map is strongly improved compared to that obtained by the PD only, but This improvement is small compared to that obtained by the segmentation of $\mathrm{T} 2$ only.

Information in GM fused map reinforced in area of agreement (mainly in the cortex). and the fusion showed a significant improvement and reduces the effect of noise in images. 
These remarks demonstrate the superior capabilities of the proposed approach compared to the taking into account of only one weighting in MR image segmentation.

The performances of our system led us to reflect on the validity of the segmentation obtained. It appeared to us to measure and quantify the performances of our segmentation of the whole of brain. Measurement used is the DSC coefficient described in section 7 and the results are reported in figures 5, 6 and 7.

The graphics of figures 5, 6 and 7 underline the advantages of the fusion of multimodality images within the fuzzy possibilistic framework to improve the results clearly. DSC coefficients obtained by the proposed approach augments the improvement of the segmentation from $2 \%$ to $3 \%$ for the white matter and from $1 \%$ to $3 \%$ for the gray matter in $\mathrm{T} 2$ image. And from $2 \%$ to $12 \%$ for the white matter and from $3 \%$ to $19 \%$ for the grey matter in PD. Image. Moreover one indeed notes that the CSF is improved only compared to the weighting PD, in that the improvement increases by $7 \%$ to $21 \%$.

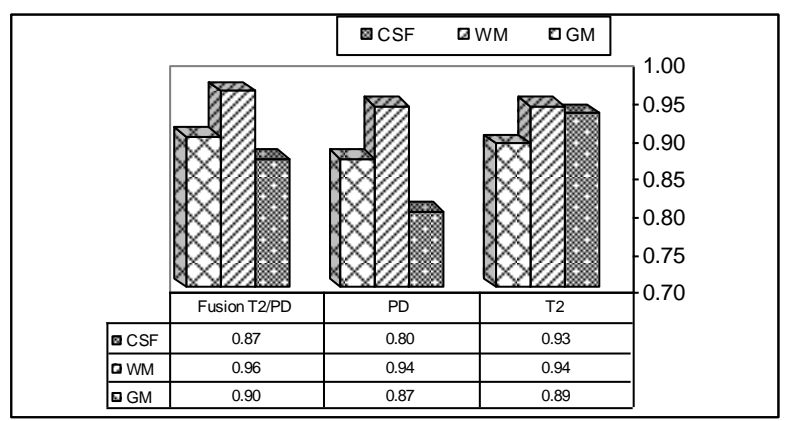

Figure 5. Comparison results between different segmentations with $0 \%$ noise

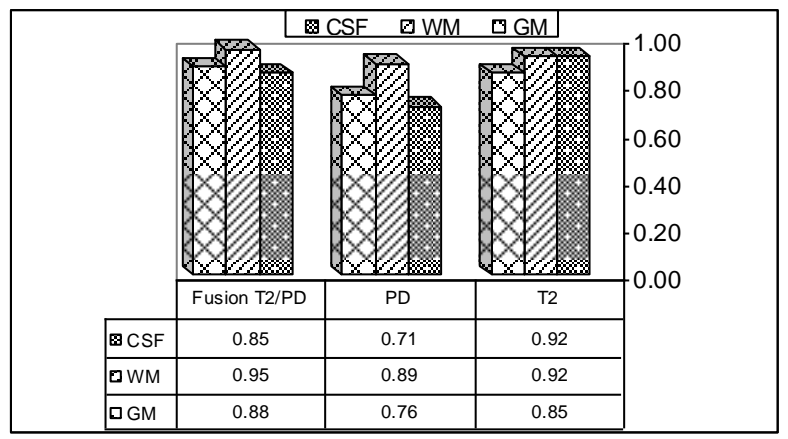

Figure 6. Comparison results between different segmentations with $3 \%$ noise

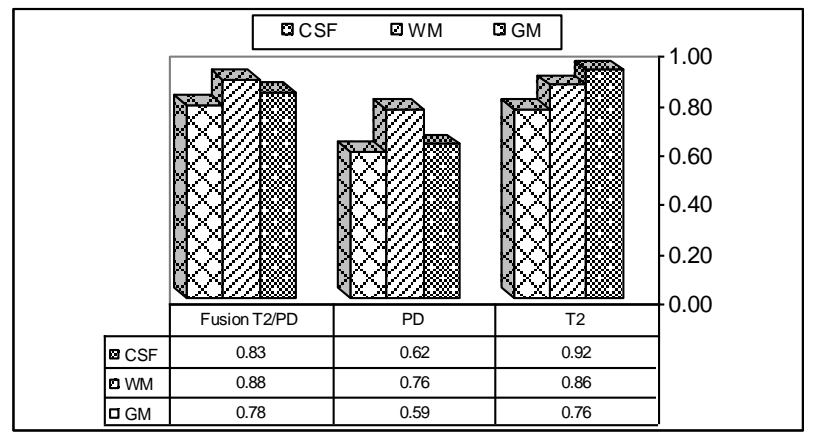

Figure 7. Comparison results between different segmentations with $5 \%$ noise

\section{CONCLUSION}

In this article we presented a system of data fusion to segment MR images in order to improve the quality of the segmentation. Since we outlined in here some features of possibility theory, which can be very useful for medical images fusion. And which constitute advantages over classical theories. They include the high flexibility of the modeling offered by possibility theory, taking into account both imprecision and uncertainty and prior information not necessarily expressed as probabilities. The Effectiveness of our system is affirmed by the choice of the model to representing data and the selected operator in a combination step. Results obtained are rather encouraging and underline the potential of the data fusion in the medical imaging field.

As a perspective of this work and on the level of modeling we would wish to integrate other information or new techniques of MR acquisitions and thus to use a more effective and more robust algorithms to representing a data. on fusion level an adaptive operators of fusion are desired for the combination of the data in order to improve the segmentation of the MR images or to detect anomalies in the pathological images.

\section{REFERENCES}

[1] I. Bloch, "Some aspects of Dempster-Shafer evidence theory for classification of multi-modality medical images taking partial volume effect into account," Pattern Recognition Letters, vol. 17, pp. 905-919, 1996.

[2] I. Bloch, and H. Maitre, "Data fusion in 2D and 3D image processing: An overview," X Brazilian symposium on computer graphics and image processing, Brazil, pp. 127-134, 1997.

[3] W. Dou, S. Ruan, Y. Chen, D. Bloyet, J. M. Constans, "A framwork of fuzzy information fusion for the segmentation of brain tumor tissues on MR images," Image and vision Computing, vol. 25, pp. 164-171, 2007

[4] V. Barra and J. Y. Boire, "A General Framework for the Fusion of Anatomical and Functional Medical Images," NeuroImage, vol. 13, 410-424, 2001.

[5] D. C. Maria, H. Valdés, J. F. Karen, M. C. Francesca, M. W. Joanna, "New multispectral MRI data fusion technique for white matter lesion segmentation: method and comparison with thresholding in FLAIR images," Eur Radiol, vol. 20, 1684-1691, 2010.

[6] E.D Waltz, the principals and practice of image and spatial data fusion in : Davis L, Hall, James llinas (Eds), Proceedings of the Eight, national Data Fusion Conference, (Dalls, TX. March 15-17, 1995) Handbook of Multisensor Data Fusion, CRC pess, West Bam Beach, FL,1995, pp. 257-278. (p4-1-4-18).

[7] F. Behloul, M. Janier, P. Croisille, C. Poirier, A. Boudraa, R. Unterreiner, J. C. Mason, D. Revel, "Automatic assessment of myocardial viability based on PET-MRI data fusion," Eng. Med. Biol. Mag., Proc. 20th Ann. Int. Conf. IEEE, vol. 1, pp. 492-495, 1998.

[8] M. Aguilar, R. Joshua, "New fusion of multi-modality volumetric medical imagery," ISIF, pp. 1206-1212, 2002.

[9] E. Lefevre, P. Vannoorenberghe, O. Colot, "About the use of Dempster-Shafer theory for color image segmentation," First international conference on color in graphics and image processing, Saint-Etienne, France, 2000.

[10] V.Barra, and J-Y Boire, "Automatic segmentation of subcortical brain structures in MR images using information fusion," IEEE Trans. on Med. Imaging., vol. 20, n7, pp. 549-558, 2001.

[11] M. C. Clarck, L.O. Hall, D.B. Goldgof, R Velthuizen, F.R. Murtagh, M.S. Silbiger, "Automatic tumor segmentation using knowledge-based techniques," IEEE Trans. Med. Imaging, vol. 17, pp. 187-201, 1998.

[12] I. Bloch, "Fusion of numerical and structural image information in medical imaging in the framework of fuzzy sets," Fuzzy Systems in Medicine, ed. par P. Szczepaniak et al., pp. 429-447, Springer Verlag, 2000. 
[13] L. A. Zadeh, "Fuzzy sets," Information and Control, vol. 8, pp. 338$353,1965$.

[14] L. A. Zadeh, "fuzzy sets as a basic for a theory of possibility," Int. Jour. of Fuzzy Sets and Systems, vol. 1, pp. 3-28, 1978.

[15] D. Dubois and H. Prade. Fuzzy sets and systems: Theory and Application, New-York: Academic Press, 1980.

[16] D. Dubois and H. Prade, "A review of Fuzzy Set Aggregation Connectives," Information Sciences, vol. 36, pp. 85-121, 1985.

[17] R. R. Yager, "Connectives and quantifiers in fuzzy sets," Int. Jour. of Fuzzy sets and systems, vol. 40, pp. 39-75,1991.

[18] D. Dubois and H. Prade, "Combination of information in the framework of possibility theory," In M. AL Abidi et al., editor, Data Fusion in Robotics and Machine Intelligence: Academic Press, 1992.

[19] V. Barra, Fusion d'images 3D du cerveau : Etude de modèles et Applications. Thèse de doctorat, Université d'Auvergne, 2000.

[20] I. Bloch, "Information combination operators for data fusion: A Comparative Review with Classification," IEEE Transactions en systems, Man. and Cybernitics, vol. 1, pp. 52-67, 1996.

[21] W. Dou, Segmentation des images multispectrales basée sur la fusion d'information: application aux images IRM. Thèse de doctorat Université CAEN/Basse-Normandi , 2006.

[22] A.Bendjebbour, W.Pieczynski, "Segmentation d'images multisenseurs par fusion évidentielle dans un contexte markovien," Traitement du Signal, vol. 14, n15, pp. 453-463, 1997.

[23] H.J. Zimmermann. Fuzzy Sets Theory and its Applications. 2nd ed. Boston: Kluwer Academic Publishers, 1991.

[24] S. Deveughelle, B. Dubuisson, "Adaptabilité et combinaison possibiliste: application à la vision multi caméras," Traitement du Signal, vol. 11, n6, pp. 559-568, 1994.

[25] C.Barillot, J.C.Gee,L.Le Briquer,G.Le Goualher, "Fusion intra et inter individus en imagerie médicale appliquée à la modélisation anatomique du cerveau humain,” Traitement du Signal, vol. 11, n6, 1994.

[26] J. Bezdek, "A convergence theorem for the fuzzy data clustering algorithms," IEEE Transactions on Pattern Analysis and Machine Intelligence TPAMI, vol. 2, pp. 1-8,1980.

[27] J. Bezdek , L. Hall, L. Clarke, "Review of MR image segmentation using pattern recognition," Medical Physics, vol. 20, pp. 1033-1048, 1993.

[28] V.Barra, J. H. Boire, Segmentation floue des tissus cérébraux en IRM
3D: une approche possibiliste versus autres méthodes, Rencontres Francophones sur la logique floue et ses applications, Valenciennes, Editions Cépaduès, pp. 193-198, 1999.

[29] I.Bloch, H. Maitre, "Fusion de données en traitement d'images : modèles d'informations et décisions," Traitement du Signal, vol. 11, n6, pp. 435-446, 1994.

[30] BrainWeb [Online]. Available: www.bic.mni.mcgill.ca/brainweb/

[31] C. A. Cocosco, V. Kollokian, R. K. -S. Kwan, and A. C. Evans, "BrainWeb: Online interface to a 3D MRI simulated brain database," NeuroImage, pt. 2/4, vol. 5, n4, p. S425, 1997.

[32] D. L. Collins, A. P. Zijdenbos, V. Kollokian, J. G. Sled, N. J. Kabani, C. J. Holmes, and A. C. Evans, "Design and construction of a realistic digital brain phantom," IEEE Trans. Med. Imaging, vol. 17, n3, pp. 463-468, 1998.

[33] I. Bloch, "Fusion d'informations en traitement du signal et des images," unpublished.

\section{AUTHORS PROFILE}

Chaabane Lamiche received his BSc in Computer Science in 1997 from the Department of Computer Science from Ferhat Abbas University, Algeria. He also received Master's degree in Computer Science in 2006 from University of M'sila. He has 4 years experience in teaching. His areas of interests include Data mining and Warehousing, artificial intelligence, Image processing and operational research. His current research interests include the data mining techniques and medical image analysis.

Abdelouahab Moussaoui received his BSc in Computer Science in 1990 from the Department of Computer Science from the University of Science and Technology of Houari Boumedienne (USTHB), Algeria. He also received and

MSc in Space Engineering in 1991 from University of Science and Technology of Oran (USTO). He received also a MSc degree in Machine Learning from Reims University (France) since 1992 and Master's degree in Computer Science in 1995 from University of Sidi Bel-abbes, Algeria and $\mathrm{PhD}$ degree in Computer Science from Ferhat Abbas University, Algeria. He is IEEE Member and AJIT, IJMMIA \& IJSC Referee. His researches are in the areas of clustering algorithms and multivariate image classification applications. His current research interests include the fuzzy neuronal network and non parametric classification using unsupervised knowledge system applied to biomedical image segmentation. He also works from a long time on pattern recognition's algorithm, complex data mining and medical image analysis. 